\title{
Mejora del sistema de evaluación de las prácticas con ordenador en la asignatura de Matemáticas Financieras I en el Grado de ADE incorporando las competencias transversales CT-3, CT-6, CT-12 y CT-13.
}

Roberto Cervelló-Royo ${ }^{\text {a }}$, Inmaculada Marques-Perez ${ }^{b}$

${ }^{a}$ Univesidad Politécnica de Valencia, Departamento de Economía y Ciencias Sociales (rocerro@upvnet.upv.es) ${ }^{b}$ Univesidad Politécnica de Valencia, Departamento de Economía y Ciencias Sociales (imarques@esp.upv.es).

\begin{abstract}
The European Higher Education Area has introduced a change in the teaching orientation, remarking a new education paradigm in the higher education, moving from a learning content to an orientation based on learning results and development of competences. The subject of Financial Mathematics includes, among others, the competences related with the learning of the contents of the subject: i) Critical synthesis of information which comes from different sources, ii) Efficient planning of work and iii) Introduction of creative solutions when solving problems. Moreover, among the different transversal competences (TC) and skills (a total of nine TCs), the following competences were choses as checkpoints: TC-12 Time planning and management and TC-13 specific tools. Under the methodological vision of action-research and the critical observation of teaching in the Financial Mathematics subject we use the lab-cases and sessions in order to propose actual cases and real-life problems; therefore, we avoid the use of simple practical exercises. By means of the IT resources and considering the need of a proper time management in the sessions, the main goal was to reach the objectives of the proposed learning. Thus, we were able to check how the solving of those practical examples in the lab-sessions, developed and reinforced several transversal competences and, in a special way, the ones taken as checkpoint.
\end{abstract}

Keywords: action-research, competences, IT resources, Financial mathematics, Time planning and management, computing tools

\section{Resumen}

El Espacio Europeo de Educación Superior ha traído consigo un cambio en la orientación de la enseñanza, marcando un nuevo paradigma de aprendizaje en la educación superior; en el que se ha pasado de un aprendizaje basado en contenidos, a una orientación basada en resultados de aprendizaje y desarrollo de competencias. La asignatura de Matemáticas financieras incluye, entre otras, las competencias relativas al aprendizaje de los contenidos de la asignatura: i) Sintetizar de forma crítica información 
proveniente de fuentes diversas, ii) Planificar eficientemente el trabajo, iii) Aportar soluciones creativas en la resolución de problemas. Además entre las muchas competencias transversales (CT) (un total de nueve CTs), se trabajaron como puntos de control las competencias CT-12_Planificación y gestión del tiempo y la CT-13 Instrumental específica. Bajo la visión metodológica de action research (investigación-acción), y ante la observación crítica de la situación de la docencia de Matemáticas Financieras se utilizaron las prácticas en laboratorio para plantear casos y problemas reales, evitando de esta manera, la mera resolución de simples ejercicios prácticos, mediante el uso y los recursos informáticos del laboratorio de prácticas, y con la necesidad de gestionar adecudamente el tiempo de las sesiones, con el fin de alcanzar los objetivos de aprendizaje propuestos. De esta manera, se pudo comprobar como con la resolución de dichos suspuestos prácticos en el aula informática, se conseguían reforzar diversas competencias transversales $y$, de manera especial, las tomadas como punto de control.

Palabras clave: action-research (investigación-acción), competencias, recursos informáticos, , matemáticas financieras, planificación y gestión del tiempo, instrumental informático

\section{Introducción}

El proceso de construcción del Espacio Europeo de Educación Superior (EEES) ha supuesto la nueva organización de las enseñanzas universitarias en sus diferentes niveles. Obligando, bajo las directrices del MEC, a la elaboración de nuevos Planes de Estudio que se han ido implantando progresivamente en la Universidad Politécnica de Valencia (UPV). Entre estos el Grado de Administración y Dirección de Empresas.

El Grado de Administración y Dirección de Empresas tiene como objetivo formar profesionales capaces de gestionar, dirigir, asesorar y evaluar las organizaciones empresariales. Los graduados tendrán una completa formación en la gestión y organización de las empresas, para poder desarrollar tareas de responsabilidad en el ámbito global de las organizaciones o en puestos directivos o intermedios de los diferentes departamentos que forman cualquier empresa (producción, recursos humanos, financiación, comercialización, inversión, administración o contabilidad).

La formación en este Grado, incluye, en los primeros cursos, contenidos de aprendizaje básicos, con carácter obligatorio, necesarios para la adquisición de conocimientos relativos a la gestión y organización de las empresas. Así, en el ámbito de las Finanzas, las Matemáticas financieras, tienen carácter obligatorio, en el primer curso del Grado, y son imprescindibles junto a la Introducción a las Finanzas, para el aprendizaje de los contenidos relativos a Economía Financiera y Dirección Financiera que se incluyen en tercero y cuarto curso, respectivamente, del Grado. La elaboración del Plan de Estudios resultante del nuevo EEES, supuso una reducción de créditos al pasar de la licenciatura al Grado, reduciéndose

(c) EY-NC-ND 2016, Universitat Politècnica de València 
un año la docencia. Esto obligó a la redistribución de los créditos de docencia, y su reducción en todas las asignaturas. También en Matemáticas Financieras.

Por otra parte, el EEES ha traído consigo un cambio en la orientación de la enseñanza, marcando un nuevo paradigma de aprendizaje en la educación superior pasando de centrarnos en el aprendizaje de contenidos, a una orientación completamente diferente basada en resultados de aprendizaje y desarrollo de competencias (Sanabria-Codesal, y otros, 2014). En la actualidad la UPV se encuentra implantando un modelo de formación en competencias transversales consideradas necesarias para el desempeño profesional de los titulados de la UPV, al margen de las competencias técnicas. Modelo que debe incorporarse en el diseño de las propuestas educativas.

La implantación del EEES impulsó, en el seno de la UPV, un debate sobre las adaptaciones y acciones a realizar por la totalidad de la comunidad universitaria para dar respuesta a los nuevos retos. Una de las líneas de adaptación al EEES ha sido la integración de las TIC en el campo de la enseñanza superior como apoyo y complemento a la enseñanza presencial, transformando el modelo de formación tradicional en la modalidad de tipo presencial. En este sentido, la UPV ha impulsado en los últimos años, a través de la iniciativa "Docencia en Red", la integración de la plataforma de aprendizaje PoliformaT. Un entorno virtual de colaboración y aprendizaje, soporte para la enseñanza presencial pero también para la impartición de cursos on-line, que incluye un conjunto muy completo de herramientas, para el suministro de material al alumno, la interacción profesor-alumno, la programación, la evaluación y la gestión de las asignaturas (Martínez Rubio, Ramírez Blanco, \& Ferrando Bataller, 2010). Herramienta incuestionable y fundamental en la docencia de cualquier materia, y elemento básico en el desarrollo de cualquier proyecto educativo.

Las investigaciones en torno a la propia práctica docente y sus consecuencias en el aprendizaje de los alumnos, son las que llevan habitualmente a las propuestas de innovación docente. La identificación de un problema específico de aprendizaje o de un dilema docente (¿hago esto o lo otro?) deben activar procesos de investigación en el aula para examinar y mejorar el aprendizaje de los alumnos (Morales, 2012). El fin último será adaptar la docencia a las circunstancias de aprendizaje, buscando el mejor resultado en el proceso de aprendizaje.

La asignatura de Matemáticas financieras, incluye entre las competencias relativas al aprendizaje de los contenidos de la asignatura, entre otras: i) Sintetitzar de forma crítica información proveniente de fuentes diversas, ii) Planificar eficientemente el trabajo, iii) Aportar soluciones creativas en la resolución de problemas. Además entre las competencias transversales, se trabajan la CT-01 Comprensión e integración, CT-02 Aplicación y pensamiento práctico, CT-03 Análisis y resolución de problemas, CT-07 Responsabilidad ética, medioambiental y profesional, CT-09 Pensamiento crítico, CT-10 Conocimiento de problemas contemporáneos, CT-11 Aprendizaje permanente, siendo punto de control de las competencias CT-12_ Planificación y gestión del tiempo y la CT-13 Instrumental específica.

(cc) EY-NC-ND 2016, Universitat Politècnica de València

Congreso In-Red (2016) 
Las sesiones de prácticas són un ámbito oportuno para el desarrollo y valoración de forma especial de algunas de estas competencias. Las prácticas informáticas se establecen expresamente en cuanto a duración y contenido en relación a los desarrollos teóricos de la asignatura, con una disponibilidad de 14 horas de prácticas informáticas en el semestre, organizadas en sesiones de dos horas, cada una, resultando un total de 7 sesiones de prácticas informáticas. Los grupos de prácticas, son numerosos (30 alumnos aprox.) y los alumnos no disponen de un ordenador para cada uno de ellos, obligando a los alumnos a compartir los recursos informáticos del aula por parejas. Por otra parte, los problemas que se plantean en las sesiones de prácticas van encaminados al cumplimiento de las competencias establecidas para el contenido teórico de la propia asignatura: sintetizar información, planificar el trabajo y resolver problemas. No pudiendo limitarse a la simple resolución de ejercicios prácticos en ordenador. Además, se hace necesario un buen aprovechamiento del tiempo en el desarrollo de las sesiones para poder abordar problemas más avanzados con el tiempo suficiente. Por otra parte, es importante contar con información relativa al grado de adquisición por el alumno, de los conocimientos que se pretenden.

\section{Objetivo}

El objetivo de la presente comunicación es presentar una propuesta de docencia que contribuye a la mejora del aprendizaje de los contenidos de la asignatura de Matemáticas Financieras, programando unas sesiones de prácticas que abordan la resolución de verdaderos problemas de finanzas, al nivel de dificultad establecido en la asignatura según sus contenidos, a partir de información y casos concretos del mercado financiero, introduciendo al alumno en el desarrollo de procesos de razonamiento más o menos complejos, y aportando al alumno herramientas de cálculo para el análisis y la resolución de problemas, así como la interpretación adecuada de los resultados. Todo ello necesario en el proceso de toma de decisiones de Gestión Financiera.

\section{Desarrollo de la innovación}

Bajo la visión metodológica de action research (investigación-acción)', y ante la observación crítica de la situación de la docencia de Matemáticas Financieras: utilizar las prácticas para el planteamiento de verdaderos problemas y no la mera resolución de ejercicios prácticos, en grupos numerosos (30 alumnos aprox.) que han de compartir los recursos informáticos del aula de prácticas, y la necesidad de gestionar adecudamente el tiempo de las sesiones para alcanzar los objetivos de aprendizaje propuestos, se plantea un proyecto de innovación docente que, resolviendo los problemas de docencia, incorpore las competencias transversales CT-6 "Trabajo en equipo y liderazgo", CT-03 "Análisis y resolución de problemas", CT-12 "Planificación y gestión del tiempo" que favorezcan un mejor proceso de aprendizaje, y CT-13 "Instrumental específica".

\footnotetext{
${ }^{1}$ Una orientación metodológica de investigación para mejorar los procesos de enseñanza-aprendizaje. Se trata de un enfoque en el que a partir de una reflexión crítica sobre la situación actual se suceden de manera cíclica fases de puesta en práctica de algún tipo de innovación y de reflexión sobre los resultados que pueda llevar a una solución práctica, en la docencia de esta asignatura.
}

(cc) EY-NC-ND 2016, Universitat Politècnica de València 


\section{Trabajo en equipo}

Los grupos de prácticas comprenden a unos 30 alumnos, y el tamaño de las aulas de prácticas obliga a los alumnos a compartir los recursos informáticos. Aprovechando esta situación, se ha optado por el trabajo en equipo para introducir de manera efectiva la competencia trasversal CT-6 "Trabajo en equipo y liderazgo".

La competencia incluye la dimensión del trabajo colaborativo o en equipo, y el desarrollo de la capacidad de liderazgo. Trabajar y liderar equipos de forma efectiva para la consecución de objetivos comunes, y contribuir al desarrollo personal y profesional de estos. Ambas están interrelacionadas, dado que el éxito del trabajo en equipo precisa de liderazgo y, por otra parte, no puede existir liderazgo si no hay equipo. Algunos autores diferencian en el trabajo en equipo distintos modelos. El modelo de trabajo cooperativo que pretende superar los conflictos y dificultades de aprendizaje debidos a las diferencia, con asistencia mutua, en las que uno de los miembros ejerce el liderazgo contribuyendo al aprendizaje de los otros, mientras que el modelo colaborativo, ayuda al estudiante a enfrentar su autonomía, no existe un líder, sino que cada uno actúa como líder en la tarea que le ha designado el grupo. Todos los integrantes del grupo son líderes y evaluadores de los conceptos que se exponen (Pastor, 2007) (Blasco Magraner \& Bernabé Valero, 2013).

Por las circunstancias del propio proceso de aprendizaje, en el contexto de enseñanzas de grado universitarias, es el modelo colaborativo el que primará: grupo con reparto de tareas equitativo, comunicación entre los miembros fluída, recomendando a los alumnos la búsqueda de sinergias y complementariedad para la conformación de grupos heterogéneos, que comprendan e interpreten los contenidos de aprendizaje de forma compartida, pero que a la vez fomente el liderazgo individual, de manera que cada miembro del grupo sea su propio líder y responda por las cuestiones que le son encomendadas por el grupo. Resultando en beneficios valiosos para el grupo, y para cada miembro, que reforzará sus conocimientos y sus argumentaciones.

Existen tres formas de poner en práctica el aprendizaje colaborativo según (Tudge, 1994) y una de ellas es la interacción de pares. Por otra parte, la conformación de los equipos de trabajo es una tarea básica. Cuando se consigue que un equipo de trabajo -lo que denominamos equipo de base- funcione, no conviene modificar su composición. Por lo tanto, una de sus principales características, es que los equipos de base sean estables y perduren a lo largo de un curso e incluso de todo el ciclo formativo (Maset, 2003). Por ello, a diferencia de años anteriores, que las parejas no eran fijas y se podían modificar en cada sesión, se ha optado por que las prácticas se realicen por parejas, formadas al inicio del curso, que se mantienen inamovibles durante todas las sesiones.

Para instruir y ayudar a los alumnos al buen desarrollo de las sesiones de aprendizaje, se realizan recomendaciones para la formación de los grupos de prácticas, destacando la importancia del compromiso y la responsabilidad para con los compañeros del equipo, para la preparación del trabajo, y para el propio desarrollo del trabajo en el aula. En la presentación de las prácticas de la asignatura, se procedió a la descripción del funcionamiento de las prácticas a lo largo del curso a través de la formación de los equipos.

(cc) EY-NC-ND 2016, Universitat Politècnica de València

Congreso In-Red (2016) 

CT-13.

Al finalizar las sesiones de prácticas, al final del curso, cada alumno se someterá a un test de evaluación, por su compañero (pareja de equipo), para que cada alumno reflexione sobre la actitud frente al compañero (Rúbrica en Anexo I). En ningún caso los resultados de las evaluaciones del trabajo en equipo se utilizarán para calificar las prácticas, solo se tendrá en cuenta la evaluación del trabajo académico realizado a lo largo del curso en las distintas sesiones. 
Tabla 1 Objetivos y Resultados de aprendizaje planteados para implementar la CT-6 "Trabajo en equipo y liderazgo"

\section{Objetivos:}

- Formar al alumnado sobre los conceptos de trabajo en equipo y liderazgo para fomentar en ellos estas capacidades.

- Concienciar sobre los tipos de liderazgo asumidos en el trabajo en equipo, y su influencia en las relaciones interpersonales y los resultados del trabajo en grupo.

- Constatar y evaluar los roles asumido por el alumnado a través de un test de liderazgo y un cuestionario de autoevaluación.

\section{Resultados de aprendizaje:}

- Participar y colaborar activamente en las tareas de equipo y fomentar la confianza, la cordialidad y la orientación a la tarea conjunta.

- Contribuir a la consolidación y desarrollo del equipo favoreciendo la comunicación, el reparto equilibrado de tareas, el clima interno y la cohesión.

- Dirigir grupos de trabajo, asegurando la integración de los miembros.

Fuente: Documento UPV “Competencias Transversales"

\section{Análisis y resolución de problemas, y Planificación y gestión del tiempo}

Las competencias, CT-03 “Análisis y resolución de problemas", CT-12 "Planificación y gestión del tiempo", y CT-13 "Instrumental específica" se implementan a través de las 7 sesiones de prácticas previstas.

Con el objeto de incorporar la competencia CT-03 "Análisis y resolución de problemas", y CT-12 "Planificación y gestión del tiempo" CT-13 Instrumental específica:

- los desarrollos prácticos en estas sesiones se basarán en problemas prácticos relativos a casos concretos que resulten de situaciones más o menos reales .

- los enunciados de los problemas deben facilitar la formación y el desarrollo de conceptos, y permitir identificar de forma clara el resultado que se busca

- $\quad$ se debe atender especialmente el procedimiento de resolución, introduciendo en el conocimiento de herramientas para dicha resolución, así como la interpretación y el análisis de los resultados de las mismas.

- deben permitir extraer conclusiones importantes para la toma de decisiones. En este sentido las aportaciones diversas que supone el trabajo en equipo resultan muy enriquecedoras

- deben de realizarse y resolverse utilizando las tecnologías más avanzadas en relación al ámbito profesional para el que se prepara el alumnoy de acuerdo con los conocimientos y las competencias profesionales que debe adquirir 


\section{Tabla 2 Objetivos y Resultados de aprendizaje planteados para implementar la CT3- "Análisis \\ y resolución de Problemas", la CT12- "Planificación y Gestión del Tiempo" y CT-13 Instrumental específica}

\section{Objetivos:}

Desarrollar en los alumnos una actitud mental mediante la aplicación de procedimientos estructurados de resolución de problemas que promueva su capacidad de aprender, comprender y aplicar conocimientos de forma autónoma

Contribuir al desarrollo de otras competencias básicas como el trabajo en equipo, la creatividad, el análisis o el liderazgo

Favorecer que el alumno incorpore en la resolución de los problemas las tareas previas de identificación, y de programación y planiciación de las actuaciones a desarrollar para la resolución de los mismos

Familiarizar al alumno en las tecnologías más avanzadas en el ámbito profesional para el que se forma

\section{Resultados de aprendizaje:}

Identificar y analizar un problema para generar alternativas de solución, aplicando los métodos aprendidos: definir con exactitud el problema a resolver, recopilar la información significativa para la solución

Utilizar la experiencia y el criterio para analizar las causas de un problema y construir una solución más eficiente y eficaz: identificar bien las causas que generan el problema, identificar las posibles alternativas para la solución, evaluar ventajas y desventajas de cada alternativa, decidir la solución oportuna, decidir la aplicación y el seguimiento de la misma

Definir claramente los objetivos a cumplir, establecer un orden de prioridades para la resolución de los mismos, y programar y planificar las actividades para la resolución

Aplicar las herramientas propias del ámbito profesional. En concreto la Hoja de cálculo, y las funciones financieras que incorpora.

Fuente: Documento UPV “Competencias Transversales”

\section{Guiónes de Prácticas}

Para trabajar la competencia CT-3, se desarrollan las siete sesiones de Prácticas, proponiendo problemas de naturaleza financiera, planteados simulando problemas de decisión a los que se enfrenta la empresa, utilizando cuando es posible datos y referencias reales del mercado financiero (tipos del mercado financiero publicadas por el Banco de España, ofertas de financiación de entidades de crédito, ...). 


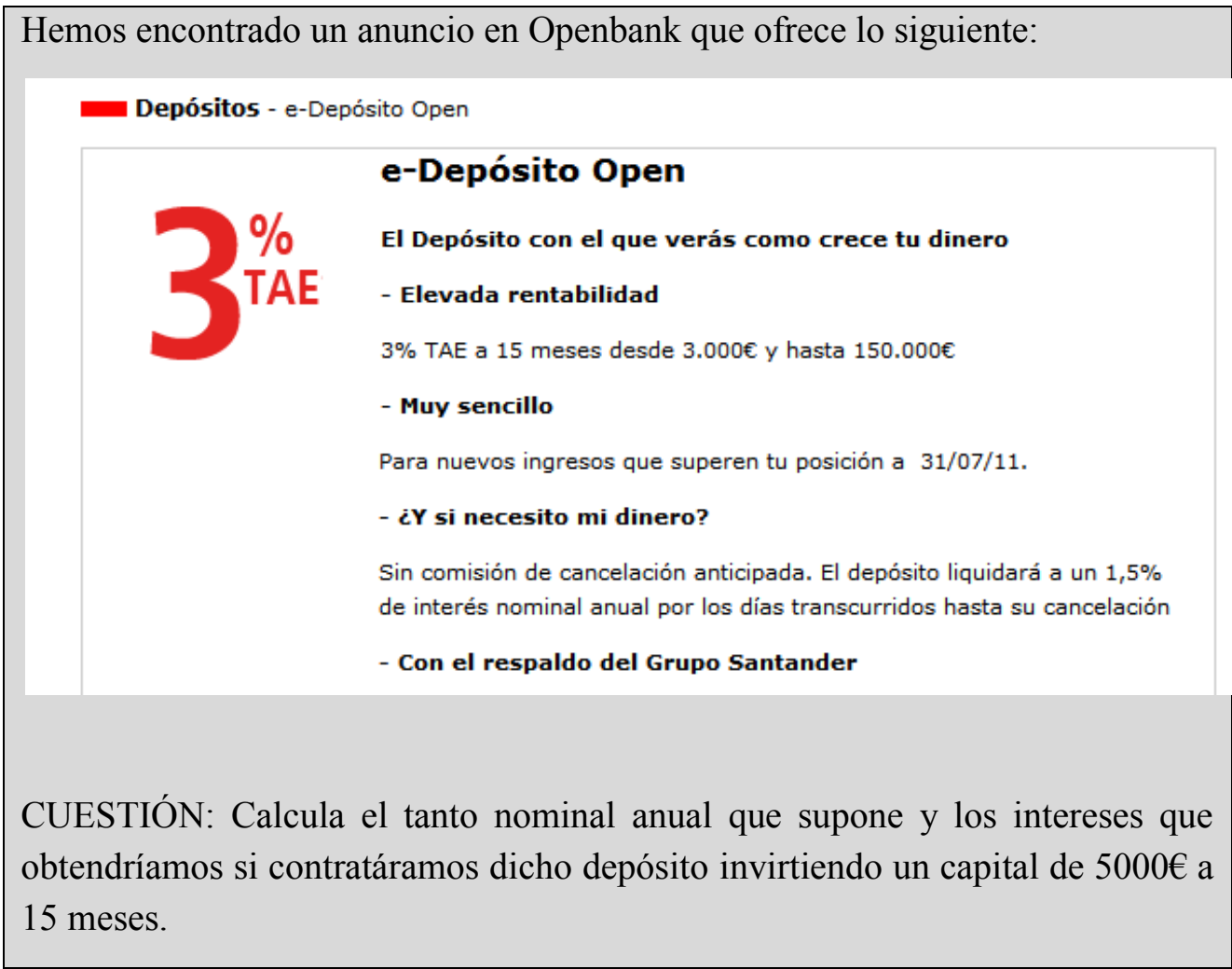

Figura 1: Ejemplo de pregunta tipo

El alumno se familiarizará así con las fuentes de datos en el sector, con el lenguaje y los conceptos, y las situaciones y problemas de carácter financiero a los que puede enfrentarse la empresa, así como con las herramientas básicas para el análisis de las mismas. El objeto es que el alumno se enfrente a la toma de decisiones relativas a alternativas de financiación, y como estudiarlas. Plantendo las prácticas en conjunto, simulando el desarrollo de las tareas de gestión de la empresa, considerando la interconexión real existente entre las distintas decisiones a tomar relativas a inversión-financiación. La utilización de las simulaciones presenta el beneficio en el aprendizaje de los alumnos, de la facilidad de comprensión de los conceptos teóricos y de los procesos a estudiar. A la vez que el aprendizaje mediante el estudio de casos permite llevar al contexto o complejidad global de la toma de decisiones, un caso particular y concreto (Álvarez y Maroto 2012), ayudando al alumno en el entendimiento del contexto global, y la interpretación de los resultados y las conclusiones que se pueden extraer en la resolución de cada problema.

Por otra parte y de acuerdo con la CT-13 la resolución de casos y supuestos se realizan con instrumental específico, en concreto hoja de cálculo y otras herramientas ofimáticas, que el alumno tendrá a su disposición en la sesión. En algunos casos, se proponen plantillas para la resolución de los problemas, a fin de ayudar al alumno a identificar los datos, las cuestiones que se plantean y la sistemática para la obtención de resultados que permitan el 
análisis, las conclusiones sobre los resultados y en definitiva la toma de decisiones a partir de los mismos.

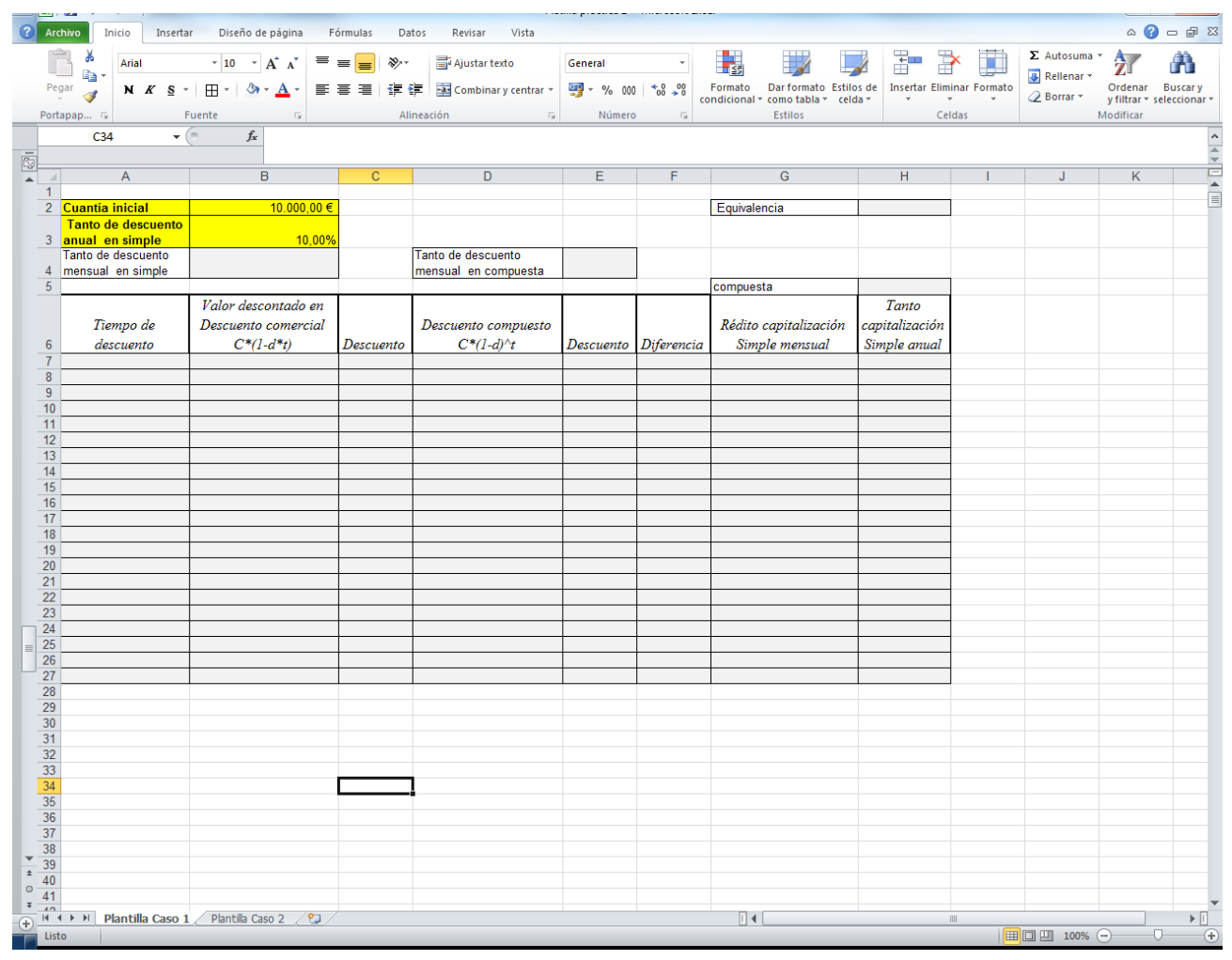

Figura 2: Ejemplo de plantillas para la identificación de datos, operaciones y resultados para el análisis y las conclusiones que permitan la toma de decisiones

Los alumnos cuentan con guiones de prácticas para cada una de las sesiones o talleres. Disponibles desde PoliformaT. En estos guiones se plantean los problemas de decisión de carácter financiero, que durante la sesión de prácticas se resolverán. En las sesiones de prácticas los grupos realizarán un trabajo tutelado por el profesor, desarrollando cada grupo sus propias herramientas informáticas para la resolución del ejercicio, en Excel. La Hoja de cálculo se archivará en el espacio compartido, desde donde estará disponible para cada alumno y para los profesores de la asignatura.

En las sucesivas sesiones el grupo irá creando su propio libro de excel, que se conformará a modo de plantilla de análisis, válida para el análisis de distintas situaciones y problemas, con el objeto de que el alumno se cree una herramienta de análisis útil para su desarrollo profesional futuro.

Como elementos de guía para el profesor, en cada práctica:

- Explicar dinámica de trabajo en cada sesión

- Lectura inicial enunciado. Repasar los conceptos vistos en las unidades. Identificación de datos y cuestiones a resolver.

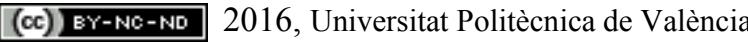


- Indicar al alumno las pautas para la elaboración-conformación de la hoja de cálculo:

- formato,

- identificación de datos,

- definición de área para los resultados,

- funciones y operaciones de cálculo a realizar

- nombrar hojas adecuadamente, ...

- Resolver dudas durante el desarrollo de la práctica

\section{Cuestionarios para la Evaluación}

La interpretación y el análisis de los resultados, para extraer conclusiones los pondrá en práctica cada alumno de forma individual al contestar un TEST al final de la sesión. El Test es de autoevaluación, generado en poliformaT, a través de una batería de preguntas que aleatoriza las preguntas y el orden de las respuestas, generando para cada alumno un test completamente distinto al de sus compañeros.

Se han elaborado baterías de aproximadamente 20 preguntas, en la plataforma. En su confección se han considerado los contenidos teóricos y los objetivos de aprendizaje de la práctica, en cuestión. Los ejercicios individuales constan de entre 5 y 10 preguntas.

Los ejercicios son autoevaluables, de manera que el alumno puede comprobar al finalizar la sesión los resultados de la evaluación, en el apartado de calificaciones, favoreciendo el feedback. Además de facilitar al profesor la tarea de evaluación.La evaluación de la competencia CT-3, se realizará con una Rubrica (Anexo II), elaborada a partir de las propuestas por la UPV. A través de las preguntas de la batería del ejercicio y la hoja de cálculo en el espacio compartido

\section{Gestión del Tiempo}

Por otra parte, la resolución de casos y supuestos se realiza con fecha límite de entrega. Normalmente la propia sesión de prácticas. La valoración de la eficiencia en la gestión del tiempo (CT-12) para la resolución de los mismos, se realiza comprobando el desarrollo de la práctica y la entrega de resultados. También se ha elaborado una Rúbrica (Anexo III).

El desarrollo del cuestionario en PoliformaT aporta datos sobre el día y la hora de entrega de la práctica, así como el tiempo empleado por cada alumno, permitiendo hacer análisis sobre el tiempo empleado por cada alumno en la resolución, y establecer comparaciones con el conjunto de los alumnos. 


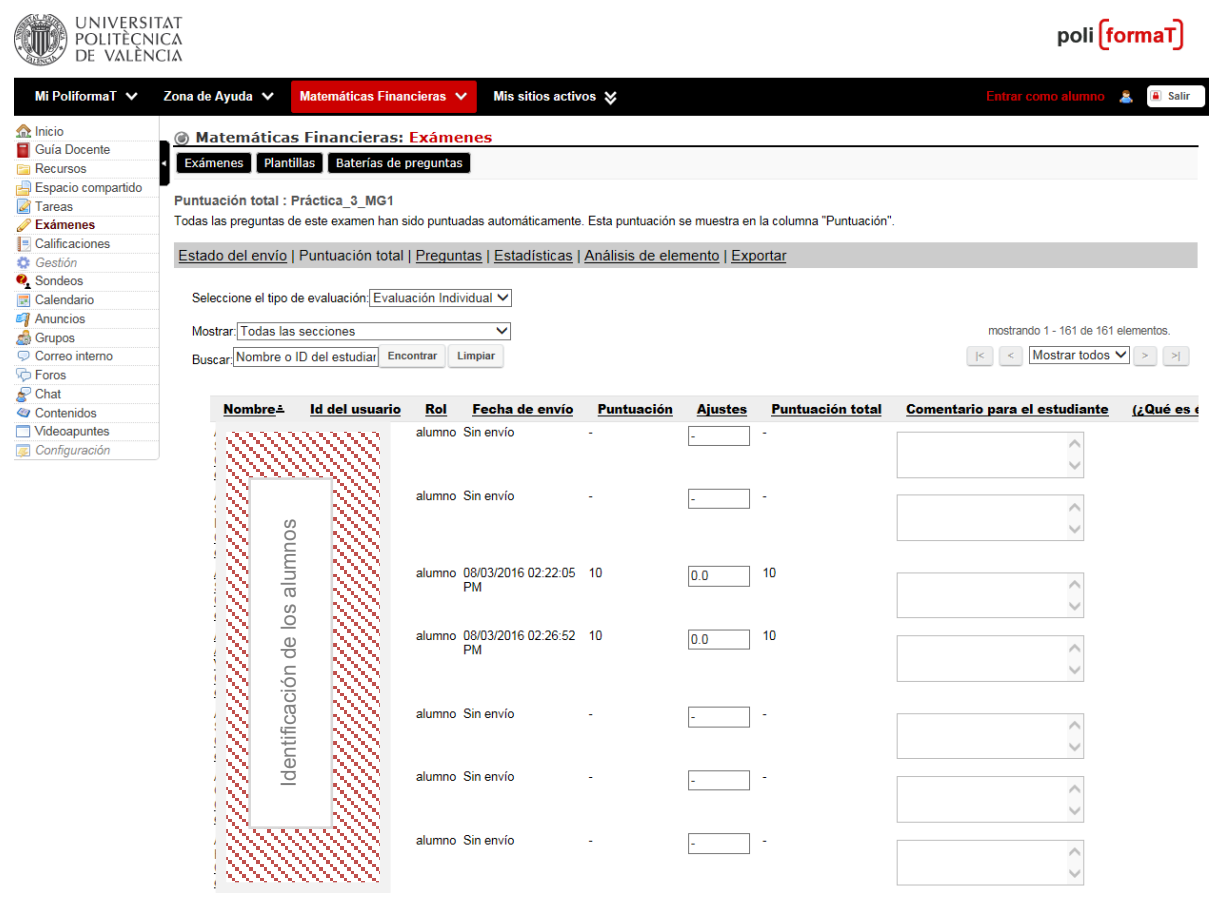

Figura 3: Datos relativos a la gestión del tiempo

\section{(C) Matemáticas Financieras: Calificaciones}

\section{Vista previa Listado Calificaciones de curso Configuración de Calific}

\section{Notas del Curso}

AVISO IMPORTANTE: La nota final del curso se calculará considerando aquella alumno. En aquella calificación en la que un alumno no tenga nota, ésta no será el botón CALCULAR NOTAS DEL CURSO que encontrará al final de esta págin

Sumario del curso

Puntos totales $\quad 300$

Nota media del curso. Notable $(78 \%)$

Tabla de evaluaciones

Sobreescriba una nota final introduciendo otra diferente en Sobreescribir Nota

Figura 4: Datos relativos a los resultados de las evaluaciones 


\section{Conclusiones}

La asignatura de Matemáticas financieras incluye, entre otras, las competencias relativas al aprendizaje de los contenidos de la asignatura: i) Sintetizar de forma crítica información proveniente de fuentes diversas, ii) Planificar eficientemente el trabajo, iii) Aportar soluciones creativas en la resolución de problemas. Además entre las competencias transversales, se trabajan la CT-01 Comprensión e integración, CT-02 Aplicación y pensamiento práctico, CT-03 Análisis y resolución de problemas, CT-07 Responsabilidad ética, medioambiental y profesional, CT-09 Pensamiento crítico, CT-10 Conocimiento de problemas contemporáneos, CT-11 Aprendizaje permanente, siendo puntos de control las competencias CT-12_ Planificación y gestión del tiempo y la CT-13 Instrumental específica. Con este fin, se plantearon seis prácticas desarrolladas para seis grupos de prácticas, desde el enfoque del caso real y haciendo uso de recursos informáticos del aula de prácticas, introduciendo al alumno en el proceso de toma de decisiones de carácter financiero, mediante dicho instrumental específico y gestionando adecudamente el tiempo de cada sesión. De esta manera, se conseguían incorporar las competencias transversales CT-6 y CT-03 al tiempo que se reforzaban de forma especial las CT puntos de control que han de propiciar un mejor proceso de aprendizaje, CT-12 "Planificación y gestión del tiempo" y CT-13 "Instrumental específica". Los altos resultados obtenidos por encima del aprobado y con una media del 78\% (sobre 100\%) (Fig 4) demuestran y avalan como las innovaciones introducidas en la asignatura, la resolución de casos reales en las prácticas de laboratorio así como el uso de las TIC y las herramientas ofimáticas bajo el prisma del action-research, consiguen reforzar diversas competencias y, de forma especial, aquellas tomadas como puntos de control, al tiempo que se obtienen mejores resultados de aprendizaje.

\section{Referencias}

ÁlVAREZ, C., \& MAROTO, J. (2012). La elección del estudio de caso en investigación educativa. Gazeta de Antropoligía, 28/1(14). http://hdl.handle.net/10481/20644.

BLASCO MAGRANER, S., \& BERNABE VALERO, G. (2013). ¿Cómo desarrollar la competencia colaborativa en el alumnado universitario? Una Propuesta de implementación $\quad y \quad$ evaluación. http://m.web.ua.es/va/ice/jornadasredes/documentos/2013-posters/335175.pdf

GUTIERREZ ESTEBAN, P., YUSTE TOSINA, R., CUBO DELGADO, S., \& LUCERO FUSTES, M. (2011). Buenas prácticas en el desarrollo de trabajo colaborativo en materias TIC aplicadas a la educación. Profesorado. Revista de currículum y formación del profesorado, 15(1). http://hdl.handle.net/10481/15367 (Consulta 3 de Abril de 2016) 
MARTINEZ RUBIO, J., RAMIREZ BLANCO, M., \& FERRANDO BATALLER, M. (2010). Docencia en Red, una apuesta estratégica en la Universidad Politécnica de Valencia. Universidad Nacional Autónoma de México. Obtenido de Reposital. Material educativo.: http://hdl.handle.net/123456789/1203 (Consulta Enero de 2016).

MASET, P. (2003). El aprendizaje cooperativo: algunas ideas prácticas. Universidad de Vic.

MORALES, P. (2012). Investigación en Innovación Educativa. Revista Iberoamericana sobre Calidad, Eficacia y Cambio en Educación, 8(2).

PASTOR, M. (2007). Ventajas del uso de la tecnología en el aprendizaje colaborativo. Revista Iberoamericana de Educación, 41(4), 5.

SANABRIA-CODESAL, E., BOSCH, I., VINCENT-VELA, M.-C., LLORET, J., ÁlVAREZ-BLANCO, S., \& ROMERO PEREZ, L. (2014). Análisis de las Dimensiones Competenciales Incluidas en Diferentes Asignaturas en Ingenierías. En U. P. Valencia (Ed.), Jornadas de Innovación Educativa y de Docencia en Red (págs. 1050-1064). Valencia: Universidad Politécnica de Valencia.

TUDGE, A. (1994). Vigotsky: la zona de desarrollo próximo y su colaboración en la práctica de aula. Universidad de Cambridge. 


\section{Anexo I}

Rúbrica para evaluar el trabajo en equipo (propuesta UPV)

\begin{tabular}{|c|c|c|c|}
\hline 1-pobre & 2- escaso & 3-bueno & 4- excelente \\
\hline \multicolumn{4}{|c|}{ CRITERIO: CONTRIBUCIÓN PARTICIPACIÓN } \\
\hline $\begin{array}{l}\text { No propongo ideas para } \\
\text { realizar el trabajo ni } \\
\text { hago sugerencias para su } \\
\text { mejora. En ocasiones } \\
\text { dificulto las propuestas } \\
\text { de otros para alcanzar } \\
\text { los objetivos grupales }\end{array}$ & $\begin{array}{l}\text { Algunas veces } \\
\text { propongo ideas para } \\
\text { realizar el trabajo pero } \\
\text { nunca hago sugerencias } \\
\text { para su mejora. Acepto } \\
\text { las propuestas de otros } \\
\text { para alcanzar los } \\
\text { objetivos del grupo }\end{array}$ & $\begin{array}{l}\text { Propongo ideas para } \\
\text { realizar el trabajo } \\
\text { aunque pocas veces } \\
\text { hago sugerencias de } \\
\text { mejora. Me esfuerzo } \\
\text { por alcanzar los } \\
\text { objetivos del grupo }\end{array}$ & $\begin{array}{l}\text { Siempre propongo } \\
\text { ideas para realizar el } \\
\text { trabajo y hago } \\
\text { sugerencias para su } \\
\text { mejora. Se esfuerza } \\
\text { para alcanzar los } \\
\text { objetivos del grupo. }\end{array}$ \\
\hline \multicolumn{4}{|l|}{ CRITERIO: ACTITUD } \\
\hline $\begin{array}{ll}\text { Muy pocas veces } \\
\text { escucho y comparto las } \\
\text { ideas de mis } \\
\text { compañeros. No ayudo a } \\
\text { mantener la unidad en el } \\
\text { grupo }\end{array}$ & $\begin{array}{l}\text { A veces escucho las } \\
\text { ideas y trato de } \\
\text { integrarlas en mis } \\
\text { propuestas aunque no } \\
\text { siempre lo hago. No } \\
\text { me preocupa la unión } \\
\text { en el grupo }\end{array}$ & $\begin{array}{l}\text { Suelo escuchar y } \\
\text { compartir las ideas de } \\
\text { mis compañeros pero } \\
\text { no siempre acepto } \\
\text { integrarlas. Colabora } \\
\text { en mantener la unión } \\
\text { en el grupo. }\end{array}$ & $\begin{array}{l}\text { Siempre escucho y } \\
\text { comparto las ideas de } \\
\text { mis compañeros e } \\
\text { intento integrarlas. } \\
\text { Busca rómo } \\
\text { mantener la unión en } \\
\text { el grupo }\end{array}$ \\
\hline \multicolumn{4}{|c|}{ CRITERIO: RESPONSABILIDAD } \\
\hline $\begin{array}{l}\text { Nunca entrego el trabajo } \\
\text { a tiempo y el grupo debe } \\
\text { modificar sus fechas o } \\
\text { plazos }\end{array}$ & $\begin{array}{l}\text { Muchas veces me } \\
\text { retraso en la entrega de } \\
\text { mi trabajo y el grupo } \\
\text { tiene que modificar a } \\
\text { veces las fechas o } \\
\text { plazos }\end{array}$ & $\begin{array}{l}\text { En ocasiones me } \\
\text { retraso en la entrega } \\
\text { del trabajo, aunque el } \\
\text { grupo no tiene que } \\
\text { modificar a veces sus } \\
\text { fechas o plazos }\end{array}$ & $\begin{array}{l}\text { Siempre entrego el } \\
\text { trabajo a tiempo y el } \\
\text { grupo no tiene que } \\
\text { modificar sus fechas } \\
\text { o plazos. }\end{array}$ \\
\hline \multicolumn{4}{|c|}{ CRITERIO: ASISTENCIA Y PUNTUALIDAD } \\
\hline $\begin{array}{l}\text { Asisto como máximo al } \\
60 \% \text { de las reuniones y } \\
\text { siempre llegó tarde }\end{array}$ & $\begin{array}{l}\text { Asisto de un } 61 \% \text { a un } \\
74 \% \text { de las reuniones y } \\
\text { no siempre llego } \\
\text { puntual }\end{array}$ & $\begin{array}{l}\text { Asisto de un } 75 \% \text { a } \\
\text { un } 90 \% \text { de las } \\
\text { reuniones y siempre } \\
\text { soy puntual }\end{array}$ & $\begin{array}{l}\text { Asisto siempre a las } \\
\text { reuniones y soy } \\
\text { puntual }\end{array}$ \\
\hline \multicolumn{4}{|c|}{ CRITERIO: RESOLUCIÓN DE CONFLICTOS } \\
\hline $\begin{array}{l}\text { No comparto los } \\
\text { recursos disponibles con } \\
\text { otros compañeros. Primo } \\
\text { mis objetivos } \\
\text { individuales frente a los } \\
\text { grupales y me cuesta } \\
\text { colaborar con otros } \\
\text { compañeros. }\end{array}$ & $\begin{array}{l}\text { En algunas ocasiones } \\
\text { comparto recursos con } \\
\text { compañeros. Me cuesta } \\
\text { pensar como un equipo } \\
\text { porque mis objetivos } \\
\text { pesan más para mí pero } \\
\text { consigo cooperar. }\end{array}$ & $\begin{array}{l}\text { Actúo como miembro } \\
\text { del equipo y no me } \\
\text { centro sólo en mis } \\
\text { propios objetivos. } \\
\text { Hago un esfuerzo (y } \\
\text { consigo) cooperar } \\
\text { con otros compañeros } \\
\text { y } \\
\text { resultados rompartir } \\
\text { quizás no aunque } \\
\text { activamente ran } \\
\text { debería. }\end{array}$ & $\begin{array}{l}\text { Siempre comparto } \\
\text { mis recursos y me } \\
\text { centro en el equipo } \\
\text { en lugar de pensar en } \\
\text { mis objetivos. } \\
\text { Colaboro activamente } \\
\text { con el resto de mis } \\
\text { compañeros. }\end{array}$ \\
\hline
\end{tabular}




\section{Anexo II}

Rúbrica para evaluar el Análisis y la Resolución de Problemas

\begin{tabular}{|c|c|c|c|c|}
\hline 1- No alcanzado & 2- En desarrollo & 3- Bien/Adecuado & 4- Excelente & EVIDENCIAS \\
\hline \multicolumn{5}{|c|}{ CRITERIO: Define el problema e identifica los datos correctamente } \\
\hline $\begin{array}{l}\text { No identifica la } \\
\text { cuestión a resolver y } \\
\text { no identifica los } \\
\text { datos }\end{array}$ & 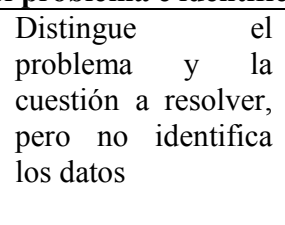 & \begin{tabular}{lr}
\multicolumn{2}{l}{ Define el problema, } \\
identificando los \\
$\begin{array}{l}\text { datos } \\
\text { relevantes }\end{array}$
\end{tabular} & $\begin{array}{l}\text { Define con exactitud } \\
\text { el problema, } \\
\text { identificando las } \\
\text { causas, sus datos e } \\
\text { incógnitas }\end{array}$ & $\begin{array}{lr}\text { Configura la } & \text { Hoja } \\
\text { adecuadamente: } & \\
\text { identificación } & \text { de } \\
\text { datos } & \\
\text { definición de áreas } \\
\text { para resultados }\end{array}$ \\
\hline \multicolumn{5}{|c|}{ CRITERIO: Emplea un método aprendido para la resolución del problema propuesto } \\
\hline $\begin{array}{l}\text { No emplea un } \\
\text { método aprendido } \\
\text { para resolver el } \\
\text { problema propuesto }\end{array}$ & $\begin{array}{lr}\text { Emplea un } & \text { método } \\
\text { aprendido } & \text { para } \\
\text { resolver el problema } \\
\text { propuesto } & \text { y } \\
\text { esquematiza } & \text { el } \\
\text { proceso } & \text { de } \\
\text { resolución } & \end{array}$ & $\begin{array}{lr}\text { Emplea un } & \text { método } \\
\text { aprendido } & \text { para } \\
\text { resolver } & \text { el } \\
\text { problema, } & \\
\text { esquematiza } & \text { el } \\
\text { proceso } & \text { de } \\
\text { resolución } & \text { y } \\
\text { justifica los cálculos } \\
\text { en cada etapa } \\
\text { (datos, ecuaciones, } \\
\text { etc.) }\end{array}$ & $\begin{array}{l}\text { Emplea un método } \\
\text { aprendido para } \\
\text { resolver el problema, } \\
\text { esquematiza el } \\
\text { proceso de resolución, } \\
\text { justifica los cálculos } \\
\text { en cada etapa (datos, } \\
\text { ecuaciones, etc.), y } \\
\text { valora su adecuación } \\
\text { (limitaciones, } \\
\text { hipótesis, etc.) }\end{array}$ & $\begin{array}{l}\text { Configuración de la } \\
\text { Hoja de Cálculo } \\
\text { Identifica } \\
\text { adecuadamente a } \\
\text { través del test de } \\
\text { PoliformaT el método } \\
\text { utilizado }\end{array}$ \\
\hline
\end{tabular}

\section{Anexo III}

\section{Rúbrica para evaluar la Planificación y Gestión del Tiempo}

\begin{tabular}{|c|c|c|c|c|}
\hline 1- No alcanzado & 2- En desarrollo & 3- Bien/Adecuado & 4- Excelente & EVIDENCIAS \\
\hline \multicolumn{5}{|c|}{ CRITERIO: Identifica las actividades a realizar en la sesión de prácticas } \\
\hline $\begin{array}{l}\text { No identifica } \\
\text { actividades en la } \\
\text { hoja de cálculo de } \\
\text { trabajo. }\end{array}$ & $\begin{array}{l}\text { Se plantea } \\
\text { actividades poco } \\
\text { concretas, } \\
\text { observándose cierta } \\
\text { confusión. }\end{array}$ & $\begin{array}{l}\text { Se plantea } \\
\text { actividades } \\
\text { concretas pero es } \\
\text { poco exigente en el } \\
\text { formato y la } \\
\text { identificación por } \\
\text { hojas. }\end{array}$ & $\begin{array}{l}\text { Organiza } \\
\text { adecuadamente las } \\
\text { actividades de la } \\
\text { práctica, en varias } \\
\text { hojas, y con formato } \\
\text { adecuado. }\end{array}$ & $\begin{array}{l}\text { Organización de la } \\
\text { Hoja de Cálculo: } \\
\text {-Utiliza varias hojas, } \\
\text { una para cada } \\
\text { actividad } \\
\text {-Nombra cada hoja } \\
\text { según la actividad } \\
\text { Completa el ejercicio. } \\
\text { realizando todos los } \\
\text { cálculos previstos, } \\
\text { contestando } \\
\text { adecuadamente el test } \\
\text { de autoevaluación }\end{array}$ \\
\hline \multicolumn{5}{|c|}{ CRITERIO: Realiza las actividades en el tiempo asignado y con el formato requerido } \\
\hline $\begin{array}{l}\text { No realiza ninguna } \\
\text { actividad en el } \\
\text { tiempo que se le ha } \\
\text { asignado }\end{array}$ & $\begin{array}{l}\text { Realiza alguna } \\
\text { actividad en el } \\
\text { tiempo asignado }\end{array}$ & $\begin{array}{l}\text { Realiza las } \\
\text { actividades en el } \\
\text { tiempo asignado, } \\
\text { pero deja por } \\
\text { contestar algunas } \\
\text { preguntas. }\end{array}$ & $\begin{array}{l}\text { Realiza las } \\
\text { actividades en el } \\
\text { tiempo asignado y no } \\
\text { deja ninguna pregunta } \\
\text { en blanco }\end{array}$ & $\begin{array}{l}\text {-Hora de inicio del } \\
\text { ejercicio, } \\
\text { comparación con } \\
\text { compañeros. } \\
\text {-Envío del alumno / } \\
\text { envío automático } \\
\text { cuando se cumple el } \\
\text { tiempo. } \\
\text { - preguntas en } \\
\quad \text { blanco. } \\
\text { - nota }\end{array}$ \\
\hline
\end{tabular}

(cc) EY-NC-ND 2016, Universitat Politècnica de València 